\title{
Wheels on Time: A Six Sigma Approach to Reduce Delay in Operating Room Starting Time
}

\author{
Anastasius O. Peter*, Arvin Parvathaneni, Cindy Wilson, Tiffany Tankalavage and Pramil Cheriyath \\ Pinnacle Health System-Harrisburg Hospital,Harrisburg, Pennsylvania,USA
}

\begin{abstract}
Delay in performing operating procedures is one of the key factors that hinders optimal flow of patients and also has a significant impact on the economic outcome of the hospital. These delays could arise at various points due to multiple personnel involved in the process of performing a surgery. The main aim of the study was to identify possible causes for delay in performing operating procedures on time and instituting effective interventions which would prevent such delays. Positive reinforcement was used to encourage the surgical teams to perform the procedures on time and avoid the delays. The mean number of cases starting on time has improved from about $10 \%$ in March 2009 to about $80 \%$ in March 2011. With the use of effective intervention strategies delay in performing operating procedures can be reduced which ultimately improves overall patient satisfaction along with improving hospital costs.
\end{abstract}

\section{Introduction}

Surgery is a collective team effort of surgeons, anesthesiologists, nurses and the paramedical staff and the delay in performing these procedures can be attributed to inept performance of these groups. Operating room delays can negatively impact patients directly, their family members and the staff. Most instances of delay are due to lack of appropriate planning or inadequate utilization of available resources. Preoperative delays are defined as delays due to system deficiencies in the operating room, which were classified in the error data base as failure of process which is delay and failure of equipment [2]. Preoperative delays hinder the optimal flow of patients and are a source of patient frustration. Several studies focused on multiple aspects of preoperative and Preoperative delays in performing the surgical procedures and tried to delineate the causes for operating room delays, so that effective interventions can be implemented to reduce these delays. Minimization of these delays with effective intervention and planning can cut down the costs of operating rooms and increase the profit margin to the hospitals [1].

One of the factors that has a significant impact on Preoperative delays is the delay in starting the first case of the day. Delayed start for the first case of the day will have a ripple effect as the following cases get delayed which result in patient annoyance and disruption of operating room and staff schedules. Avoiding the delay in first case would not only be beneficial from a financial perspective but the quality of patient care, appropriate utilization of staff time and resources available are improved. This study focused exclusively on the delay in starting the first case each day in the operating rooms of Harrisburg hospital and Community general hospital. The advisory committee has set the national bench mark of $59 \%$ for the number of first cases being wheeled in on time in each operating room. This cutoff mark is for the hospitals who do not accept the 5 minute grace period policy and for those who have the 5 minute grace period policy the bench mark has been set at $72 \%$. In March 2009 the percentage of first cases being wheeled in on time was $13 \%$ in Harrisburg hospital and $6 \%$ in Community general. Both the hospitals were significantly below the appropriate marks setup by the advisory committee. The intervention method designed to be used in the study was the lean six sigma approach [4].

Six sigma is a widely approved interventional strategy which was originally designed by Motorola in 1986. This approach measures quality in terms of defect rates and keeps the target error rate to less than 3.4 million opportunities [5]. After its success it has been applied in several different industries to improve the productivity and minimize the variability in outcomes. Six sigma process employs DMAIC (Define, Measure, Analyze, Improve, Control) and DMADV
(Define, Measure, Analyze, Design, Verify) two different approaches in designing new interventions. The six sigma process assigns hierarchy to the people involved in designing and monitoring the intervention process throughout the study [3].

\section{Methods}

\section{Define the problem}

The national advisory committee has set the bench mark of the percentage of first cases being wheeled in as 59\% in operating rooms across United States. Before the project was implemented the rate of first cases being wheeled in on time was $13 \%$ at Harrisburg hospital and $7 \%$ at Community general hospital. The lag in the rate from national bench mark was a result of interplay of various factors in the operating room. The two main factors identified for the delays were

1. surgeon arrival time

2. Patient flow in the pre-op area.

The delays were quantified at different levels and the following are the potential targets for improvement.

1. Patient wait time from the time of arrival to the time they are taken to pre-op area is 28 minutes.

2. Only $50 \%$ of the time the pre-op RN's were done with getting the patients ready for surgery.

3. Half of the surgeons were arriving after 7:28 (median time) to see the patients before OR while the first case of the day was supposed to begin at 7:30 am (Figure 1).

\section{Project Comittee}

Lean Six Sigma projects comprise of administrative champions

*Corresponding author: Dr. Anastasius O Peter, 117 Lake Point Dr HARRISBURG PA17111, USA, Tel: 417693 1662; Fax: 717724 8535; E-mail: panastasius@ pinnaclehealth.org

Received October 20, 2011; Accepted November 17, 2011; Published November 19, 2011

Citation: Peter AO, Parvathaneni A, Wilson C, Tankalavage T, Cheriyath P (2011) Wheels on Time: A Six Sigma Approach to Reduce Delay in Operating Room Starting Time. Surgery 1:102. doi:10.4172/2161-1076.1000102

Copyright: (C) 2011 Peter AO, et al. This is an open-access article distributed under the terms of the Creative Commons Attribution License, which permits unrestricted use, distribution, and reproduction in any medium, provided the original author and source are credited. 
who are accountable for the success of the project. They are the heavy lifters who make sure system issues don't block the change efforts. Sue Comp along with Dr Wisman and Dr Kellis were the champions. Cyndy Wilson was the Black Belt (lean six sigma terminology for project manager and facilitator of change) who was assigned to this project. The OR Committee played the role of the steering committee which collaboratively made all the decisions related to this project. Dr Raymond Kostin (the Chair) \& Dr Ronald Lippe (the Co-Chair) led the committee members (who were Surgeons, Anesthesiologists and Surgical Services Administration) to a successful outcome with this project. And the patient is in the center of all the activity as it is because of the patient that we are all here.

\section{Definition of terms}

An important initiative in starting this project was to achieve an acceptable definition of what the 7:30 start time on the OR schedule represented. 118 internal customers with representation from both sites and all the roles (surgeons, anesthesiologists, surgical services staff) were surveyed. The question asked was what does start time mean? Does it mean wheels in the room, induction time, incision time, what is the standard everyone is using to tell time -clocks on the wall, computers? Watches? cell phones? were all those times the same? One of the questions they were asked was what time should patients be wheeled in the room for an OR scheduled time of 7:30. The chart below depicts the responses (Figure 2).

Based on the responses obtained from different personnel in the operating room the OR committee made the decision to standardize the time for wheels in to be 7:25 (and 7:55 on Wednesdays). After deciding the new timelines the baseline data showed only $9 \%$ of the First OR cases at $\mathrm{HH}$ and 6\% at CGH were "Wheel in Time". Here are two definitions that were used to standardize delays

Surgeon Delay is documented in Pathfinder when the Circulating Nurse goes over to the Pre-Op area by 7:20 and they are unable to take the patient to the OR because the surgeon has not finished their part in time for "wheels in" to occur by 7:25 (7:55 on Wednesdays).

Anesthesia Delay is documented in Pathfinder when the Circulating Nurse goes over to the Pre-Op area by 7:20 and they are unable to take the patient to the OR because the anesthesiologist has not finished their part in time for "wheels in" to occur by 7:25 (7:55 on Wednesdays).

\section{Process}

The success of a team is achieved when each and every team is held accountable for his or her own actions and the progress of the team. In order to promote a culture of accountability the following steps were followed

1. Team agreement form was developed

2. Interlocking accountability among the team members was promoted

3. Transparency in the data was increased

4. Promote team member buy in

5. Identify rewards and consequences to help modify the behavior

A common agreement was formulated for all the team members to agree on and adhere to in order to improve the percentage of wheels in on time first cases of the day. The agreements were printed as huge posters and were placed in three different areas of the OR so that there would be an opportunity for everyone to see it. The OR committee tried to blend in accountability through team agreement so that it helped define each member's role and responsibilities. It also established clear standards of excellence for team members. After the surgical team started following the time agreement the delays were monitored closely and the decrease in the number of delays was evident. The whole concept of working together as a team as opposed to separate individuals was a critical component of the whole project. If the team was successful in achieving the proposed targets then each member of the team received gold star as an incentive for their success. If the delay could not be avoided then all the team members received nothing (Figure 5).

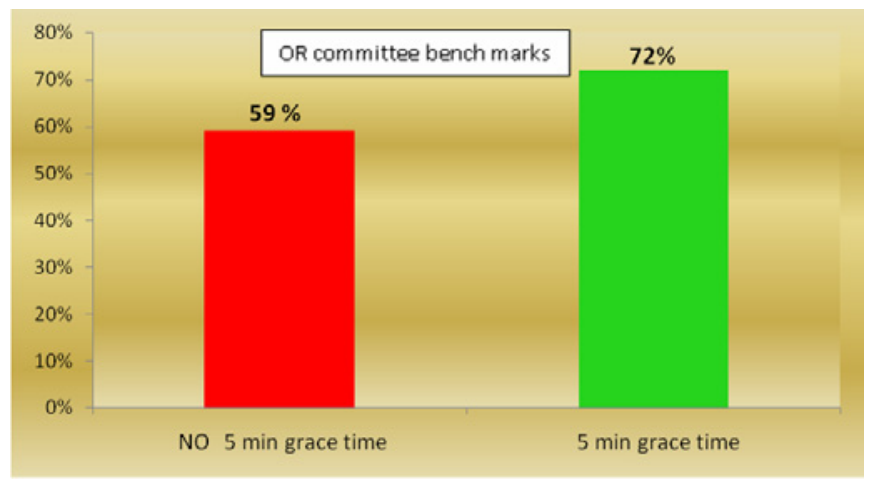

Figure 1:

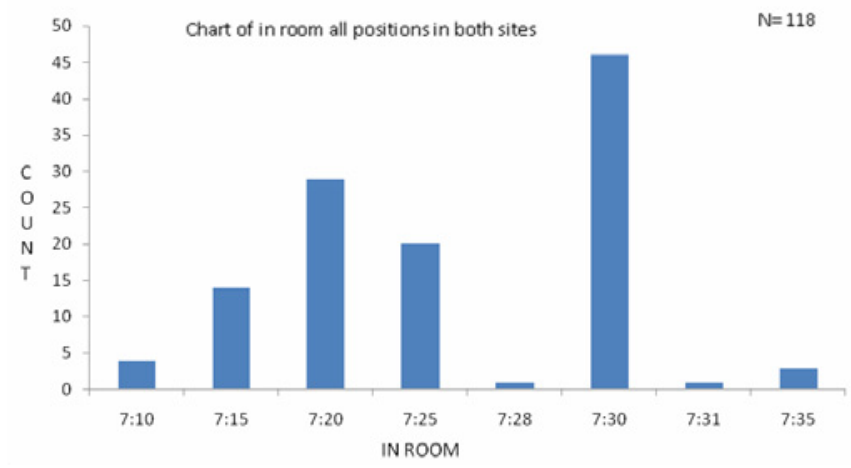

Figure 2:

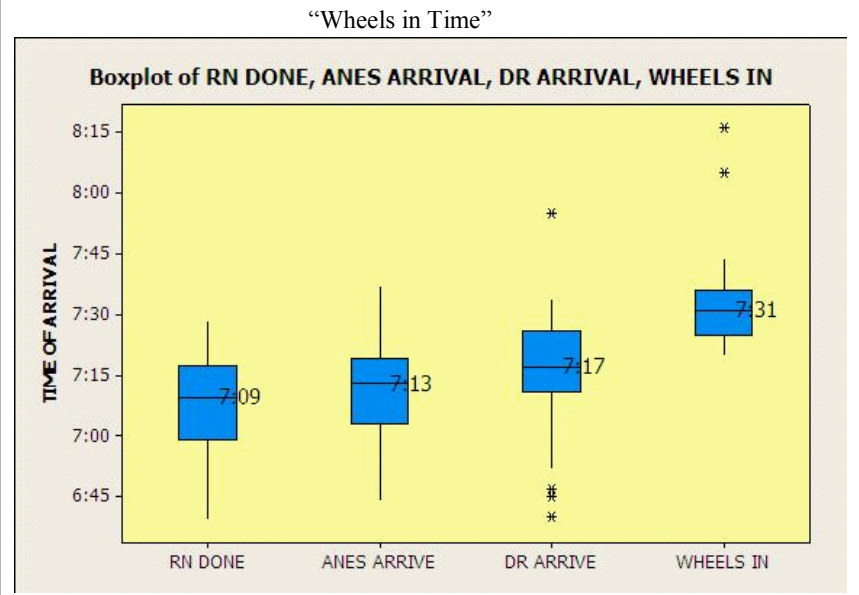

Figure 3: 
In October 2009 a brief review of the project was performed to monitor the progress due to changes implemented by the project. The time when the Pre-Op RN was done, Surgeon and Anesthesiologist arrival times and the resulting Wheels In time were documented each day of the week except Wednesday for the whole month of October. The Wheels In time was 7:31, which means each of the team members needed to complete their respective parts sooner to achieve the 7:25 am goal. The Pre-op RN done time stamp was added to be an accountability and communication tool because it defined the end of pre-op RN's part of the wheels in time process. The goal was set to be achieved by 7:10 $90 \%$ of the time. It also communicates to the OR nurses, surgeons, anesthesiologists, CRNA's and other members of the team that patient is ready for the next step. As a result of this process change the patients were getting to the pre-op bay 14 minutes sooner. By January 2010 the pre-op RN's first met their goal of $90 \%$ as a result of various process changes (Figure 3 ).

\section{Results}

The data was collected over the period of 2 years from march- 09 to march-11. The data was stratified based on delays caused by each member of the team and was arranged by each week and month. These delays were recorded by using a standardized recording system based on the definitions devised by the project committee. The following graph depicts the total number of first cases wheeled in on time each day of the week as per the regulations devised by the OR committee.

The six sigma methodology used in this project yielded significant increase in the number of first cases being wheeled in on time. At Harrisburg hospital the first cases being wheeled in on time increased from 13\% in March 2009 before the project to 80\% in March 2011. Similarly at community general hospital the increase in the number of cases was from 7\% in March 2009 to $83 \%$ in March 2011. The data collected was quantified based on delays caused by different members of the team. Significant changes were observed in the main concerns addressed at the start of the study which were the surgeon delay and the delay caused in the pre-op area. The pre-op nurse completing on time has improved from $50 \%$ of the cases to about $80 \%$ of cases in Harrisburg hospital and $83 \%$ of the cases in Community general hospital. The delays caused due to arrival of surgeon in the operating room have gone down from $13 \%$ in Community general and $9 \%$ at Harrisburg hospital in March 2010 to 1\% in both the hospitals in

\section{RESPONSE TO THE INTERVENTION}

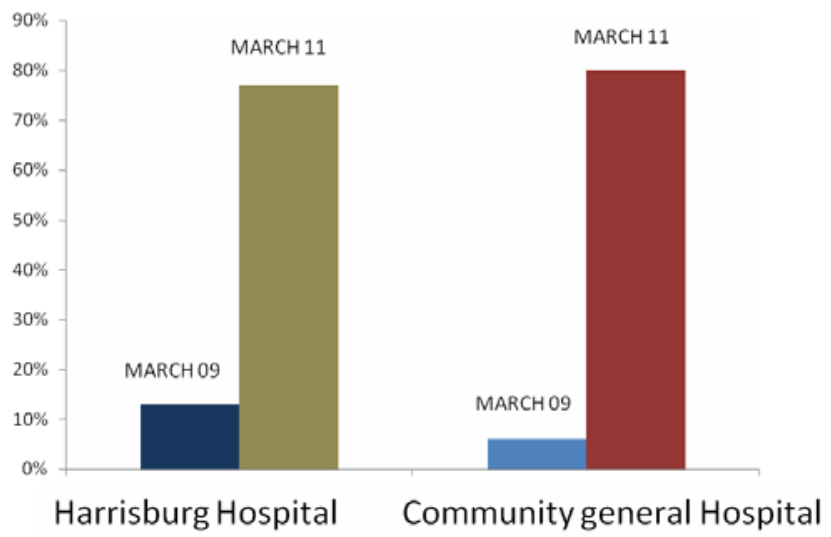

Figure 4:

\section{TEAM AGREEMENT "Wheels In Time"}
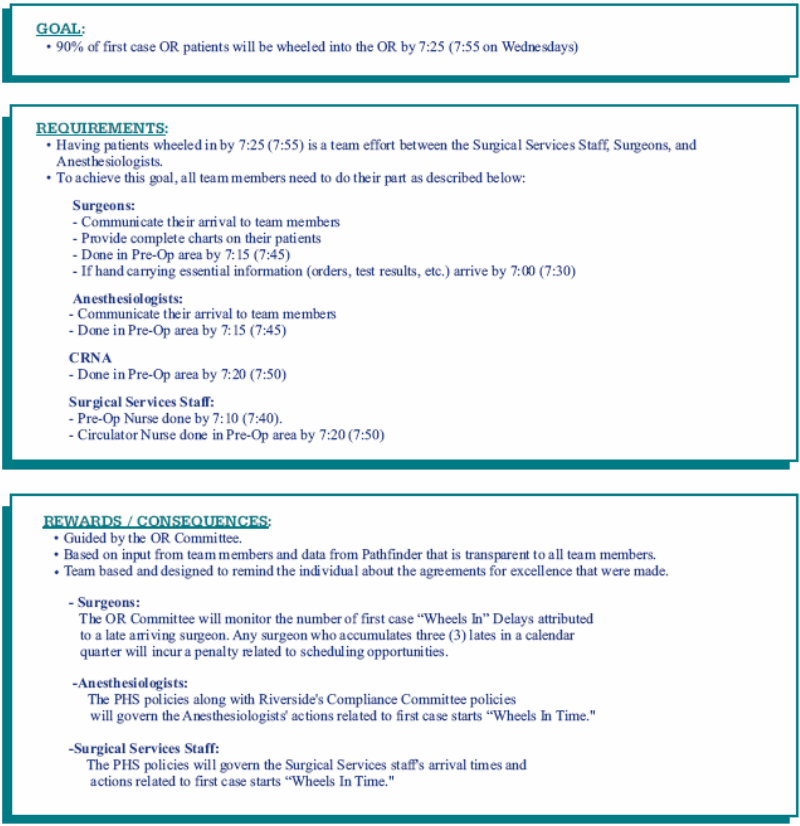

This Agreement finalized by the PinnacleHealth System OR Committee on 12-23-09.

PINNACLEHEALTH $\triangleleft>$ Proven.

Figure 5:

March 2011. The delays caused by patients have gone down from $7 \%$ to $1 \%$ in Community general and from $5 \%$ to $3 \%$ in Harrisburg hospital (Figure 4)

\section{Discussion}

The project was initiated with the goal of increasing the number of first cases of the day being wheeled in on time which would result in

1. Preventing delays for the rest of the scheduled cases for the day

2. Improve effective utilization of OR time which is cost effective

3. Improve patient satisfaction

A successful approach like six sigma intervention has been chosen to improve the efficiency of functioning of the surgical team. The analyses of the results have shown that the approach has been successful in achieving the anticipated purpose. The primary factors hindering the target were identified and appropriate interventions were designed and were monitored. Periodic reviews were performed to assess the progress and also the need for further interventions to achieve the target successfully. The multidisciplinary approach of the entire surgical team has been the key reason for the success of the project. These kinds of projects can be implemented in operating rooms in various hospitals across the country which will enhance their efficiency. In addition to improving efficiency the costs involved in maintaining the operating rooms and performing the procedures can be reduced and the margin of profit for the hospitals can be increased substantially. Focus should be laid on other possible approaches to achieve the above mentioned goals in more effective ways possible. 
Citation: Peter AO, Parvathaneni A, Wilson C, Tankalavage T, Cheriyath P (2011) Wheels on Time: A Six Sigma Approach to Reduce Delay in Operating Room Starting Time. Surgery 1:102. doi:10.4172/2161-1076.1000102

\section{References}

1. Harders.M, Malangoni.AM, Weight.S, Sidhu.T (2006) Improving operating room efficiency through process redesign. Journal of surgery 140: 509-516.

2. Wong.J, Khu.KJ, Kaderali.Z, Bernstein.M (2010) Delays in the operating room: signs of an imperfect system. Canadian journal of surgery 53: 189-195.
3. Fischman.D (2010) Applying lean six sigma methodologies to improve efficiency, timeliness of care and quality of care in an internal medicine residency clinic. Quality management in health care journal 19: 201-210

4. Operating rooms efficiency project. Six sigma black belt report: operating room process improvement. 2009 University of Michigan, College of engineering.

5. Starting the first surgical case on time to cut delays. www.isixsigma.com 